Journal of Mathematics and Informatics

Vol. 16, 2019, 87-95

ISSN: 2349-0632 (P), 2349-0640 (online)

Published 23 April 2019

www.researchmathsci.org

DOI: http://dx.doi.org/10.22457/jmi.131av16a8

Journal of

Mathematics and

Informatics

\title{
Weakly Semi-Compatible Maps and Fixed Points in Non- Archimedean Menger PM-Space
}

\author{
V.K. Gupta ${ }^{1}$, Arihant Jain ${ }^{2}$ and Rambabu Dangi ${ }^{1}$ \\ ${ }^{I}$ Department of Mathematics, Govt. Madhav Science P.G. College \\ Ujjain (M.P.) India \\ ${ }^{2}$ School of Studies in Mathematics, Vikram University \\ Ujjain (M.P.) India \\ ${ }^{2}$ Corresponding author. Email: arihant2412@gmail.com \\ Received 1 March 2019; accepted 20 April 2019
}

Abstract. This paper introduces the notion of weakly semi-compatible self-maps in a Menger PM-space and establishes a fixed point theorem for six self-maps. Our result generalizes and extends the result of Cho et al. [2] as well as Sharma et al. [14].

Keywords: Non-Archimedean Menger probabilistic metric space, Common fixed points, Compatible maps, Weakly semi-compatible maps

AMS Mathematics Subject Classification (2010): 47H10, 54H 25

\section{Introduction}

There have been a number of generalizations of metric space. One such generalization is Menger space initiated by Menger [10]. It is a probabilistic generalization in which we assign to any two points $\mathrm{x}$ and $\mathrm{y}$, a distribution function $\mathrm{F}_{\mathrm{x}, \mathrm{y}}$. Schweizer and Sklar [12] studied this concept and gave some fundamental results on this space.

The notion of compatible mapping in a Menger space has been introduced by Mishra [11]. Using the concept of compatible mappings of type (A), Jain et al. [4, 5] proved some interesting fixed point theorems in Menger space. Afterwards, Jain et al. [6] proved the fixed point theorem using the concept of weak compatible maps in Menger space.

The notion of non-Archimedean Menger space has been established by Istr a tescu and Crivat [9]. The existence of fixed point of mappings on non-Archimedean Menger space has been given by Istr ă tescu [8]. This has been the extension of the results of Sehgal and Bharucha-Reid [13] on a Menger space. Cho. et al. [2] proved a common fixed point theorem for compatible mappings in non-Archimedean Menger PM-space. In the sequel, Singh et al. [16] established the fixed point theorem for six self maps and an example using the concept of semi-compatible self maps in a non-Archimedean Menger PM-space.

In this paper, we generalize and extend the result of Cho et al. [2] and Sharma et. al. [14] by introducing the notion of semi-compatible self maps. Also, we cited an example in support of this. 


\section{V .K. Gupta, Arihant Jain and Rambabu Dangi}

\section{Preliminaries}

For terminologies, notations and properties of Menger PM-space, refer to $[1,8,15]$.

Definition 2.1. [2] Let $X$ be a non-empty set and $\mathcal{D}$ be the set of all left-continuous distribution functions. An ordered pair $(X, F)$ is called a non-Archimedean probabilistic metric space (briefly, a N.A. PM-space) if $f$ is a mapping from $X \times X$ into $\mathcal{D}$ satisfying the following conditions (the distribution function $f(x, y)$ is denoted by $F_{x, y}$ for all $x, y$ $\in \mathrm{X})$ :

(PM-1) $\mathrm{F}_{\mathrm{u}, \mathrm{v}}(\mathrm{x})=1$, for all $\mathrm{x}>0$, if and only if $\mathrm{u}=\mathrm{v}$;

$(\mathrm{PM}-2) \mathrm{F}_{\mathrm{u}, \mathrm{v}}=\mathrm{F}_{\mathrm{v}, \mathrm{u}}$;

$(\mathrm{PM}-3) \mathrm{F}_{\mathrm{u}, \mathrm{v}}(0)=0$;

(PM-4) If $\mathrm{F}_{\mathrm{u}, \mathrm{v}}(\mathrm{x})=1$ and $\mathrm{F}_{\mathrm{v}, \mathrm{w}}(\mathrm{y})=1$ then $\mathrm{F}_{\mathrm{u}, \mathrm{w}}(\max \{\mathrm{x}, \mathrm{y}\})=1$,

for all $\mathrm{u}, \mathrm{v}, \mathrm{w} \in \mathrm{X}$ and $\mathrm{x}, \mathrm{y}>0$.

Definition 2.2. [2] A t-norm is a function $\Delta:[0,1] \times[0,1] \rightarrow[0,1]$ which is associative, commutative, non-decreasing in each coordinate and $\Delta(\mathrm{a}, 1)=\mathrm{a}$ for every $\mathrm{a} \in[0,1]$.

Definition 2.3. [2] A N.A. Menger PM-space is an ordered triple (X, $\mathrm{f}, \Delta)$, where $(\mathrm{X}, \mathrm{f})$ is a non-Archimedean $\mathrm{PM}$-space and $\Delta$ is a t-norm satisfying the following condition:

(PM-5) $\quad \mathrm{F}_{\mathrm{u}, \mathrm{w}}(\max \{\mathrm{x}, \mathrm{y}\}) \geq \Delta\left(\mathrm{F}_{\mathrm{u}, \mathrm{v}}(\mathrm{x}), \mathrm{F}_{\mathrm{v}, \mathrm{w}}(\mathrm{y})\right)$, for all $\mathrm{u}, \mathrm{v}, \mathrm{w} \in \mathrm{X}$ and $\mathrm{x}, \mathrm{y} \geq 0$.

Definition 2.4. [2] A PM-space $(X, F)$ is said to be of type $(C)_{g}$ if there exists a $\mathrm{g} \in \Omega$ such that

$$
\mathrm{g}\left(\mathrm{F}_{\mathrm{x}, \mathrm{y}}(\mathrm{t})\right) \leq \mathrm{g}\left(\mathrm{F}_{\mathrm{x}, \mathrm{z}}(\mathrm{t})\right)+\mathrm{g}\left(\mathrm{F}_{\mathrm{z}, \mathrm{y}}(\mathrm{t})\right)
$$

for all $\mathrm{x}, \mathrm{y}, \mathrm{z} \in \mathrm{X}$ and $\mathrm{t} \geq 0$, where $\Omega=\{\mathrm{g} \mid \mathrm{g}:[0,1] \rightarrow[0, \infty)$ is continuous, strictly decreasing, $g(1)=0$ and $g(0)<\infty\}$.

Definition 2.5. [2] A N.A. Menger PM-space $(X, f, \Delta)$ is said to be of type (D) $)_{g}$ if there exists a $\mathrm{g} \in \Omega$ such that

$$
\mathrm{g}(\Delta(\mathrm{s}, \mathrm{t}) \leq \mathrm{g}(\mathrm{s})+\mathrm{g}(\mathrm{t})
$$

for all $\mathrm{s}, \mathrm{t} \in[0,1]$.

Remark 2.1. [2]

(1) If a N.A. Menger PM-space $(X, f, \Delta)$ is of type (D) $)_{g}$ then $(X, F, \Delta)$ is of type $(C)_{g}$.

(2) If a N.A. Menger PM-space $(X, F, \Delta)$ is of type (D) $)_{g}$, then it is metrizable, where the metric $\mathrm{d}$ on $\mathrm{X}$ is defined by

$d(x, y)=\int_{0}^{1} g\left(F_{x, y}(t)\right) d(t)$ for all $x, y \in X$. 
Weakly Semi-Compatible Maps and Fixed Points in Non-Archimedean Menger

PM-Space

Throughout this paper, suppose $(X, F, \Delta)$ be a complete N.A. Menger PM-space of type (D) $)_{\mathrm{g}}$ with a continuous strictly increasing t-norm $\Delta$.

Let $\phi:[0,+\infty) \rightarrow[0, \infty)$ be a function satisfied the condition $(\Phi)$ :

(Ф) $\quad \phi$ is upper-semicontinuous from the right and $\phi(t)<\mathrm{t}$ for all $\mathrm{t}>0$.

Lemma 2.1. [2] If a function $\phi:[0,+\infty) \rightarrow[0,+\infty)$ satisfies the condition $(\Phi)$, then we have

(1) For all $\mathrm{t} \geq 0, \lim _{\mathrm{n} \rightarrow \infty} \phi^{\mathrm{n}}(\mathrm{t})=0$, where $\phi^{\mathrm{n}}(\mathrm{t})$ is $\mathrm{n}$-th iteration of $\phi(\mathrm{t})$.

(2) If $\left\{t_{n}\right\}$ is a non-decreasing sequence of real numbers and $t_{n+1} \leq \phi\left(t_{n}\right), n=1,2$, $\ldots$ then $\lim _{n \rightarrow \infty} t_{n}=0$. In particular, if $t \leq \phi(t)$ for all $t \geq 0$, then $t=0$.

Definition 2.6. [2] Let $A, S: X \rightarrow X$ be mappings. $A$ and $S$ are said to be compatible if $\lim _{n \rightarrow \infty} g\left(F_{A S x_{n}}, S A x_{n}(t)\right)=0$ for all $t>0$, whenever $\left\{x_{n}\right\}$ is a sequence in $X$ such that $\lim _{n \rightarrow \infty} A x_{n}=\lim _{n \rightarrow \infty} S x_{n}=z$ for some $z$ in $X$.

Definition 2.7. [16] Let $A, S: X \rightarrow X$ be mappings. $A$ and $S$ are said to be semicompatible if $\lim _{n \rightarrow \infty} g\left(F_{A S x_{n}, S z}(t)\right)=0$ for all $t>0$, whenever $\left\{x_{n}\right\}$ is a sequence in $X$ such that $\lim _{\mathrm{n} \rightarrow \infty} \mathrm{Ax}_{\mathrm{n}}=\lim _{\mathrm{n} \rightarrow \infty} \mathrm{Sx}_{\mathrm{n}}=\mathrm{z}$ for some $\mathrm{z}$ in $\mathrm{X}$.

Definition 2.8. Let $A, S: X \rightarrow X$ be mappings. A and $S$ are said to be weakly semicompatible if $\lim _{n \rightarrow \infty} \mathrm{g}\left(\mathrm{F}_{\mathrm{ASX}_{\mathrm{n}}, \mathrm{Sz}}(\mathrm{t})\right)=0$ or $\lim _{\mathrm{n} \rightarrow \infty} \mathrm{g}\left(\mathrm{F}_{\mathrm{SAx}_{\mathrm{n}}, \mathrm{Az}}(\mathrm{t})\right)=0$ for all $\mathrm{t}>0$, whenever $\left\{\mathrm{x}_{\mathrm{n}}\right\}$ is a sequence in $X$ such that $\lim _{n \rightarrow \infty} A x_{n}=\lim _{n \rightarrow \infty} S x_{n}=z$ for some $z$ in $X$.

Clearly, semi-compatible maps are weakly semi-compatible maps but converse is not true.

Definition 2.9. [15] Self maps $A$ and $S$ of a N.A. Menger PM-space (X, $f, \Delta)$ are said to be weakly compatible (or coincidentally commuting) if they commute at their coincidence points, i.e. if $\mathrm{Ap}=\mathrm{Sp}$ for some $\mathrm{p} \square \in \mathrm{X}$ then $\mathrm{ASp}=\mathrm{SAp}$.

Remark 2.2. [15] Compatible maps are weakly compatible but converse is not true.

Definition 2.10. [14] Self maps $A$ and $S$ of a N.A. Menger PM-space (X, $F, \Delta$ ) are said to be occasionally weakly compatible (owc) if and only if there is a point $\mathrm{x}$ in $\mathrm{X}$ which is coincidence point of $\mathrm{A}$ and $\mathrm{S}$ at which $\mathrm{A}$ and $\mathrm{S}$ commute.

Remark 2.3. [16] The concept of semi-compatibility is more general than that of compatibility.

Lemma 2.2. [2] Let A, B, S, T:X $\rightarrow X$ be mappings satisfying the condition (1) and (2) as follows : 
V .K. Gupta, Arihant Jain and Rambabu Dangi

(1) $\quad \mathrm{A}(\mathrm{X}) \subset \mathrm{T}(\mathrm{X})$ and $\mathrm{B}(\mathrm{X}) \subset \mathrm{S}(\mathrm{X})$.

(2) $\quad g\left(F_{A x, B y}(t)\right) \leq \phi\left(\max \left\{g\left(F_{S x, T y}(t)\right), g\left(F_{S x, A x}(t)\right), g\left(F_{T y, B y}(T)\right)\right.\right.$, $\left.\left.1 / 2\left(\mathrm{~g}\left(\mathrm{~F}_{\mathrm{Sx}, \mathrm{By}}(\mathrm{T})\right)+\mathrm{g}\left(\mathrm{F}_{\mathrm{Ty}, \mathrm{Ax}}(\mathrm{t})\right)\right)\right\}\right)$

for all $\mathrm{t}>0$, where a function $\phi:[0,+\infty) \rightarrow[0,+\infty)$ satisfies the condition $(\Phi)$. Then the sequence $\left\{\mathrm{y}_{\mathrm{n}}\right\}$ in $\mathrm{X}$, defined by $\mathrm{Ax}_{2 \mathrm{n}}=\mathrm{Tx}_{2 \mathrm{n}+1}=\mathrm{y}_{2 \mathrm{n}}$ and $\mathrm{Bx}_{2 \mathrm{n}+1}=\mathrm{Sx}_{2 \mathrm{n}+2}=\mathrm{y}_{2 \mathrm{n}+1}$ for $\mathrm{n}=$ $0,1,2, \ldots$, such that

$\lim _{n \rightarrow \infty} g\left(F_{y_{n}, y_{n+1}}(t)\right)=0$ for all $t>0$ is a Cauchy sequence in $X$.

\section{Main result}

Theorem 3.1. Let A, B, S, T, L, M : X $\rightarrow X$ be mappings satisfying the condition

$\mathrm{L}(\mathrm{X}) \subset \mathrm{ST}(\mathrm{X}), \mathrm{M}(\mathrm{X}) \subset \mathrm{AB}(\mathrm{X})$;

$\mathrm{AB}=\mathrm{BA}, \mathrm{ST}=\mathrm{TS}, \mathrm{LB}=\mathrm{BL}, \mathrm{MT}=\mathrm{TM}$; either $\mathrm{AB}$ or $\mathrm{L}$ is continuous;

(L, AB) is weakly semi-compatible and (M, ST) is occasionally weakly compatible;

$$
\mathrm{g}\left(\mathrm{F}_{\mathrm{Lx}, \mathrm{My}}(\mathrm{t})\right) \leq \phi\left(\operatorname { m a x } \left\{\mathrm{g}\left(\mathrm{F}_{\mathrm{ABx}, \mathrm{STy}}(\mathrm{t})\right), \mathrm{g}\left(\mathrm{F}_{\mathrm{ABx}, \mathrm{Lx}}(\mathrm{t})\right), \mathrm{g}\left(\mathrm{F}_{\mathrm{STy}, \mathrm{My}}(\mathrm{t})\right),\right.\right.
$$

for all $\mathrm{t}>0$, where a function $\phi:[0,+\infty) \rightarrow[0,+\infty)$ satisfies the condition $(\Phi)$.

Then A, B, S, T, L and M have a unique common fixed point in X.

Proof: Let $\mathrm{x}_{0} \in \mathrm{X}$. From condition (3.1.1) $\exists \mathrm{x}_{1}, \mathrm{x}_{2} \in \mathrm{X}$ such that

$$
\mathrm{Lx}_{0}=\mathrm{STx}_{1}=\mathrm{y}_{0} \text { and } \mathrm{Mx}_{1}=\mathrm{ABx}_{2}=\mathrm{y}_{1} \text {. }
$$

Inductively, we can construct sequences $\left\{\mathrm{x}_{\mathrm{n}}\right\}$ and $\left\{\mathrm{y}_{\mathrm{n}}\right\}$ in $\mathrm{X}$ such that

(3.1.6) $\quad \mathrm{Lx}_{2 \mathrm{n}}=\mathrm{STx}_{2 \mathrm{n}+1}=\mathrm{y}_{2 \mathrm{n}}$ and $\mathrm{Mx}_{2 \mathrm{n}+1}=\mathrm{ABx}_{2 \mathrm{n}+2}=\mathrm{y}_{2 \mathrm{n}+1}$ for $\mathrm{n}=0,1,2, \ldots$

Step 1. We prove that $\lim _{n \rightarrow \infty} g\left(F_{y_{n}, y_{n+1}}(t)\right)=0$ for all $t>0$.

From (3.1.5) and (3.1.6), we have

$$
\begin{aligned}
& \mathrm{g}\left(\mathrm{F}_{\mathrm{y}_{2 \mathrm{n}}, \mathrm{y}_{2 \mathrm{n}+1}}(\mathrm{t})\right)=\mathrm{g}\left(\mathrm{F}_{\mathrm{Lx}_{2 \mathrm{n}}, \mathrm{Mx} \mathrm{x}_{2 \mathrm{n}+1}}(\mathrm{t})\right)
\end{aligned}
$$

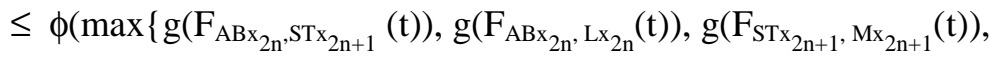

$$
\begin{aligned}
& \left.\left.1 / 2\left(g\left(F_{A B x_{2 n}, M x_{2 n+1}}(t)\right)+g\left(F_{S T x_{2 n+1}, L_{2 n}}(t)\right)\right)\right\}\right) \\
& =\phi\left(\operatorname { m a x } \left\{\mathrm{g}\left(\mathrm{F}_{\mathrm{y}_{2 n-1}, \mathrm{y}_{2 \mathrm{n}}}(\mathrm{t})\right), \mathrm{g}\left(\mathrm{F}_{\mathrm{y}_{2 \mathrm{n}-1}, \mathrm{y}_{2 \mathrm{n}}}(\mathrm{t})\right), \mathrm{g}\left(\mathrm{F}_{\mathrm{y}_{2 \mathrm{n}}, \mathrm{y}_{2 \mathrm{n}+1}}(\mathrm{t})\right)\right.\right. \text {, } \\
& \left.\left.1 / 2\left(g\left(F_{y_{2 n-1},} y_{2 n+1}(t)\right)+g(1)\right)\right\}\right) \\
& \leq \phi\left(\operatorname { m a x } \left\{g\left(\mathrm{~F}_{\mathrm{y}_{2 n-1}, \mathrm{y}_{2 \mathrm{n}}}(\mathrm{t})\right), \mathrm{g}\left(\mathrm{F}_{\mathrm{y}_{2 \mathrm{n}}, \mathrm{y}_{2 \mathrm{n}+1}}(\mathrm{t})\right)\right.\right. \text {, } \\
& \left.1 / 2\left(g\left(F_{y_{2 n-1}}, y_{2 n}(t)\right)+g\left(F_{y_{2 n}, y_{2 n+1}}(t)\right)\right\}\right) \text {. }
\end{aligned}
$$

If $g\left(F_{y_{2 n-1}, y_{2 n}}(t)\right) \leq g\left(F_{y_{2 n}, y_{2 n+1}}(t)\right)$ for all $t>0$, then by (3.1.5)

$$
\mathrm{g}\left(\mathrm{F}_{\mathrm{y}_{2 \mathrm{n}}, \mathrm{y}_{2 \mathrm{n}+1}}(\mathrm{t})\right) \leq \phi\left(\mathrm{g}\left(\mathrm{F}_{\mathrm{y}_{2 \mathrm{n}}, \mathrm{y}_{2 \mathrm{n}+1}}(\mathrm{t})\right)\right)
$$

on applying Lemma 2.1, we have $g\left(\mathrm{~F}_{\mathrm{y}_{2 n}, \mathrm{y}_{2 \mathrm{n}+1}}(\mathrm{t})\right)=0$ for all $\mathrm{t}>0$.

Similarly, we have $\mathrm{g}\left(\mathrm{F}_{\mathrm{y}_{2 \mathrm{n}+1}, \mathrm{y}_{2 \mathrm{n}+2}}(\mathrm{t})\right)=0$ for all $\mathrm{t}>0$. 
Weakly Semi-Compatible Maps and Fixed Points in Non-Archimedean Menger PM-Space

Thus, we have

$$
\lim _{n \rightarrow \infty} g\left(F_{y_{n}, y_{n+1}}(t)\right)=0 \text { for all } t>0 .
$$

On the other hand, if $g\left(\mathrm{~F}_{\mathrm{y}_{2 \mathrm{n}-1}, \mathrm{y}_{2 \mathrm{n}}}(\mathrm{t})\right) \geq \mathrm{g}\left(\mathrm{F}_{\mathrm{y}_{2 \mathrm{n}}, \mathrm{y}_{2 \mathrm{n}+1}}(\mathrm{t})\right)$, then by (3.1.5), we have

$$
g\left(F_{y_{2 n}, y_{2 n+1}}(t)\right) \leq \phi\left(g\left(F_{y_{2 n-1}, y_{2 n}}(t)\right)\right) \text { for all } t>0 \text {. }
$$

Similarly, $\quad g\left(F_{y_{2 n+1}, y_{2 n+2}}(t)\right) \leq \phi\left(g\left(F_{y_{2 n}, y_{2 n+1}}(t)\right)\right)$ for all $t>0$.

Thus, we have $\mathrm{g}\left(\mathrm{F}_{\mathrm{y}_{\mathrm{n}}, \mathrm{y}_{\mathrm{n}+1}}(\mathrm{t})\right) \leq \phi\left(\mathrm{g}\left(\mathrm{F}_{\mathrm{y}_{\mathrm{n}-1}, \mathrm{y}_{\mathrm{n}}}(\mathrm{t})\right)\right)$ for all $\mathrm{t}>0 \& \mathrm{n}=1,2,3, \ldots$

Therefore, by Lemma 2.1,

$$
\lim _{n \rightarrow \infty} g\left(F_{y_{n}, y_{n+1}}(t)\right)=0 \text { for all } t>0 \text {, which implies that }\left\{y_{n}\right\} \text { is a Cauchy sequence }
$$
in $\mathrm{X}$ by Lemma 2.2.

Since $(X, f, \Delta)$ is complete, the sequence $\left\{y_{n}\right\}$ converges to a point $\mathrm{z} \in \mathrm{X}$. Also its subsequences converges as follows :

$$
\begin{aligned}
& \left\{\mathrm{Mx}_{2 \mathrm{n}+1}\right\} \rightarrow \mathrm{z} \text { and } \quad\left\{\mathrm{STx}_{2 \mathrm{n}+1}\right\} \rightarrow \mathrm{z}, \\
& \left\{\mathrm{Lx}_{2 \mathrm{n}}\right\} \rightarrow \mathrm{z} \text { and } \quad\left\{\mathrm{ABx}_{2 \mathrm{n}}\right\} \rightarrow \mathrm{z} .
\end{aligned}
$$

Case I. $\mathrm{AB}$ is continuous.

As $\mathrm{AB}$ is continuous, $(\mathrm{AB})^{2} \mathrm{x}_{2 \mathrm{n}} \rightarrow \mathrm{ABz}$ and $(\mathrm{AB}) \mathrm{Lx}_{2 \mathrm{n}} \rightarrow \mathrm{ABz}$.

As $(\mathrm{L}, \mathrm{AB})$ is weakly semi-compatible, so $\mathrm{L}(\mathrm{AB}) \mathrm{x}_{2 \mathrm{n}} \rightarrow \mathrm{ABz}$.

Step 2. Putting $x=A B x_{2 n}$ and $y=x_{2 n+1}$ for $t>0$ in (3.1.5), we get

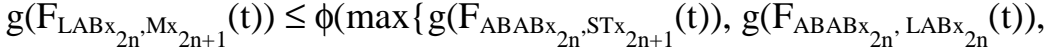

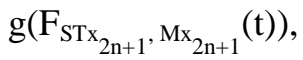

$$
\begin{aligned}
& \left.\left.1 / 2\left(g\left(F_{A B A B x}, M_{2 n}(t)\right)+g\left(F_{S T x_{2 n+1}}, \operatorname{LABx}_{2 n}(t)\right)\right)\right\}\right) .
\end{aligned}
$$

Letting $\mathrm{n} \rightarrow \infty$, we get

$$
\begin{aligned}
& \mathrm{g}\left(\mathrm{F}_{\mathrm{ABz}, \mathrm{z}}(\mathrm{t})\right) \leq \phi\left(\operatorname { m a x } \left\{\mathrm{g}\left(\mathrm{F}_{\mathrm{ABz}, \mathrm{z}}(\mathrm{t})\right), \mathrm{g}\left(\mathrm{F}_{\mathrm{ABz}, \mathrm{ABz}}(\mathrm{t})\right), \mathrm{g}\left(\mathrm{F}_{\mathrm{z}, \mathrm{z}}(\mathrm{t})\right),\right.\right. \\
&\left.\left.1 / 2\left(\mathrm{~g}\left(\mathrm{~F}_{\mathrm{ABz}, \mathrm{z}}(\mathrm{t})\right)+\mathrm{g}\left(\mathrm{F}_{\mathrm{z}, \mathrm{ABz}}(\mathrm{t})\right)\right)\right\}\right) \\
&=\phi\left(\mathrm{g}\left(\mathrm{F}_{\mathrm{ABz}, \mathrm{z}}(\mathrm{t})\right)\right)
\end{aligned}
$$

which implies that $\mathrm{g}\left(\mathrm{F}_{\mathrm{ABz}, \mathrm{z}}(\mathrm{t})\right)=0$ by Lemma 2.1 and so we have $\mathrm{ABz}=\mathrm{z}$.

Step 3. Putting $x=z$ and $y=x_{2 n+1}$ for $t>0$ in (3.1.5), we get

$$
\begin{aligned}
& \mathrm{g}\left(\mathrm{F}_{\mathrm{Lz}, \mathrm{Mx}} \mathrm{x}_{2 \mathrm{n}+1}(\mathrm{t})\right) \leq \phi\left(\operatorname { m a x } \left\{\mathrm{g}\left(\mathrm{F}_{\mathrm{ABz}, \mathrm{ST} \mathrm{S}_{2 \mathrm{n}+1}}(\mathrm{t})\right), \mathrm{g}\left(\mathrm{F}_{\mathrm{ABz}, \mathrm{Lz}}(\mathrm{t})\right), \mathrm{g}\left(\mathrm{F}_{\mathrm{STx}_{2 \mathrm{n}+1}, \mathrm{Mx}_{2 \mathrm{n}+1}}(\mathrm{t})\right),\right.\right. \\
& \left.\left.1 / 2\left(\mathrm{~g}\left(\mathrm{~F}_{\mathrm{ABz}, \mathrm{Mx}_{2 \mathrm{n}+1}}(\mathrm{t})\right)+\mathrm{g}\left(\mathrm{F}_{\mathrm{STx}_{2 \mathrm{n}+1}, \mathrm{Lz}}(\mathrm{t})\right)\right)\right\}\right) \text {. }
\end{aligned}
$$

Letting $\mathrm{n} \rightarrow \infty$, we get

$$
\begin{aligned}
\mathrm{g}\left(\mathrm{F}_{\mathrm{Lz}, \mathrm{Z}}(\mathrm{t})\right) \leq & \phi\left(\operatorname { m a x } \left\{\mathrm{g}\left(\mathrm{F}_{\mathrm{z}, \mathrm{z}}(\mathrm{t})\right), \mathrm{g}\left(\mathrm{F}_{\mathrm{z}, \mathrm{Lz}}(\mathrm{t})\right), \mathrm{g}\left(\mathrm{F}_{\mathrm{z}, \mathrm{z}}(\mathrm{t})\right),\right.\right. \\
\left.\left.1 / 2\left(\mathrm{~g}\left(\mathrm{~F}_{\mathrm{z}, \mathrm{z}}(\mathrm{t})\right)+\mathrm{g}\left(\mathrm{F}_{\mathrm{z}, \mathrm{Lz}}(\mathrm{t})\right)\right)\right\}\right) & \\
= & \phi\left(\mathrm{g}\left(\mathrm{F}_{\mathrm{L} z, \mathrm{z}}(\mathrm{t})\right)\right)
\end{aligned}
$$

which implies that $g\left(F_{L z, z}(t)\right)=0$ by Lemma 2.1 and so we have $L z=z$.

Therefore, $\mathrm{ABz}=\mathrm{Lz}=\mathrm{z}$.

Step 4. Putting $x=B z$ and $y=x_{2 n+1}$ for $t>0$ in (3.1.5), we get 
V .K. Gupta, Arihant Jain and Rambabu Dangi

$$
\begin{aligned}
& \mathrm{g}\left(\mathrm{F}_{\mathrm{LBz}, \mathrm{Mx}} \mathrm{n}_{2 \mathrm{n}+1}(\mathrm{t})\right) \leq \phi\left(\operatorname { m a x } \left\{\mathrm{g}\left(\mathrm{F}_{\mathrm{ABB} z, \mathrm{STx}} \mathrm{z}_{2 \mathrm{n}+1}(\mathrm{t})\right), \mathrm{g}\left(\mathrm{F}_{\mathrm{ABBz}, \mathrm{LBz}}(\mathrm{t})\right), \mathrm{g}\left(\mathrm{F}_{\mathrm{STx}_{2 \mathrm{n}+1}, \mathrm{Mx}_{2 \mathrm{n}+1}}(\mathrm{t})\right),\right.\right. \\
& \left.\left.1 / 2\left(\mathrm{~g}\left(\mathrm{~F}_{\mathrm{ABBz}, \mathrm{Mx}_{2 \mathrm{n}+1}}(\mathrm{t})\right)+\mathrm{g}\left(\mathrm{F}_{\mathrm{STx}_{2 \mathrm{n}+1}, \mathrm{LBz}}(\mathrm{t})\right)\right)\right\}\right)
\end{aligned}
$$

As $B L=L B, A B=B A$, so we have

$$
\mathrm{L}(\mathrm{Bz})=\mathrm{B}(\mathrm{Lz})=\mathrm{Bz} \text { and } \mathrm{AB}(\mathrm{Bz})=\mathrm{B}(\mathrm{ABz})=\mathrm{Bz} \text {. }
$$

Letting $\mathrm{n} \rightarrow \infty$, we get

$$
\begin{aligned}
\mathrm{g}\left(\mathrm{F}_{\mathrm{Bz}, \mathrm{Z}}(\mathrm{t})\right) \leq \phi\left(\operatorname { m a x } \left\{\mathrm{g}\left(\mathrm{F}_{\mathrm{Bz}, \mathrm{z}}(\mathrm{t})\right), \mathrm{g}\left(\mathrm{F}_{\mathrm{Bz}, \mathrm{Bz}}(\mathrm{t})\right), \mathrm{g}\left(\mathrm{F}_{\mathrm{z}, \mathrm{z}}(\mathrm{t})\right),\right.\right. & \left.\left.1 / 2\left(\mathrm{~g}\left(\mathrm{~F}_{\mathrm{Bz}, \mathrm{z}}(\mathrm{t})\right)+\mathrm{g}\left(\mathrm{F}_{\mathrm{z}, \mathrm{Bz}}(\mathrm{t})\right)\right)\right\}\right) \\
& =\phi\left(\mathrm{g}\left(\mathrm{F}_{\mathrm{Bz}, \mathrm{Z}}(\mathrm{t})\right)\right)
\end{aligned}
$$

which implies that $\mathrm{g}\left(\mathrm{F}_{\mathrm{Bz}, \mathrm{Z}}(\mathrm{t})\right)=0$ by Lemma 2.1 and so we have $\mathrm{Bz}=\mathrm{z}$. Also, $\mathrm{ABz}=\mathrm{z}$ and so $\mathrm{Az}=\mathrm{z}$.

(3.1.9) Therefore, $\quad \mathrm{Az}=\mathrm{Bz}=\mathrm{Lz}=\mathrm{z}$.

Step 5. As $L(X) \subset S T(X)$, there exists $v \in X$ such that $z=L z=S T v$.

Putting $x=x_{2 n}$ and $y=v$ for $t>0$ in (3.1.5), we get

$$
\begin{aligned}
& \mathrm{g}\left(\mathrm{F}_{\mathrm{Lx}_{2 \mathrm{n}}, \mathrm{Mv}}(\mathrm{t})\right) \leq \phi\left(\operatorname { m a x } \left\{\mathrm{g}\left(\mathrm{F}_{\mathrm{ABx}_{2 \mathrm{n}}, \mathrm{STv}}(\mathrm{t})\right), \mathrm{g}\left(\mathrm{F}_{\mathrm{ABx}_{2 \mathrm{n}}, \mathrm{Lx} x_{2 \mathrm{n}}}(\mathrm{t})\right), \mathrm{g}\left(\mathrm{F}_{\mathrm{STv}, \mathrm{Mv}}(\mathrm{t})\right)\right.\right. \text {, } \\
& \left.\left.1 / 2\left(\mathrm{~g}\left(\mathrm{~F}_{\mathrm{ABx}} \mathrm{x}_{2 \mathrm{n}}, \mathrm{Mv}(\mathrm{t})\right)+\mathrm{g}\left(\mathrm{F}_{\mathrm{STv}, \mathrm{Lx}_{2 \mathrm{n}}}(\mathrm{t})\right)\right)\right\}\right) .
\end{aligned}
$$

Letting $\mathrm{n} \rightarrow \infty$ and using equation (3.1.8), we get

$$
\begin{aligned}
\mathrm{g}\left(\mathrm{F}_{\mathrm{z}, \mathrm{Mv}}(\mathrm{t})\right) & \leq \phi\left(\operatorname { m a x } \left\{\mathrm{g}\left(\mathrm{F}_{\mathrm{z}, \mathrm{z}}(\mathrm{t})\right), \mathrm{g}\left(\mathrm{F}_{\mathrm{z}, \mathrm{z}}(\mathrm{t})\right), \mathrm{g}\left(\mathrm{F}_{\mathrm{z}, \mathrm{Mv}}(\mathrm{t})\right),\right.\right. \\
\left.\left.1 / 2\left(\mathrm{~g}\left(\mathrm{~F}_{\mathrm{z}, \mathrm{Mv}}(\mathrm{t})\right)+\mathrm{g}\left(\mathrm{F}_{\mathrm{z}, \mathrm{z}}(\mathrm{t})\right)\right)\right\}\right) & \\
& =\phi\left(\mathrm{g}\left(\mathrm{F}_{\mathrm{z}, \mathrm{Mv}}(\mathrm{t})\right)\right)
\end{aligned}
$$

which implies that $\mathrm{g}\left(\mathrm{F}_{\mathrm{z}, \mathrm{Mv}}(\mathrm{t})\right)=0$ by Lemma 2.1 and so we have $\mathrm{z}=\mathrm{Mv}$.

Hence, $S T v=z=M v$, which shows that $v$ is a coincidence point of ST and M.

As (M, ST) is occasionally weakly compatible, we have

$$
\text { STMv }=\text { MSTv. }
$$

Thus, $\mathrm{STz}=\mathrm{Mz}$.

Step 6. Putting $\mathrm{p}=\mathrm{x}_{2 \mathrm{n}}, \mathrm{q}=\mathrm{z}$ for $\mathrm{t}>0$ in (3.1.5), we get

$$
\begin{aligned}
& \mathrm{g}\left(\mathrm{F}_{\mathrm{Lx}_{2 \mathrm{n}}, \mathrm{Mz}}(\mathrm{t})\right) \leq \phi\left(\operatorname { m a x } \left\{\mathrm{g}\left(\mathrm{F}_{\mathrm{ABx}_{2 \mathrm{n}}, \mathrm{STz}}(\mathrm{t})\right), \mathrm{g}\left(\mathrm{F}_{\mathrm{ABx}_{2 \mathrm{n}}, \mathrm{Lx} 2 \mathrm{n}}(\mathrm{t})\right), \mathrm{g}\left(\mathrm{F}_{\mathrm{STz}, \mathrm{Mz}}(\mathrm{t})\right)\right.\right. \text {, } \\
& \left.\left.1 / 2\left(g\left(F_{A B x_{2 n}}, M_{z}(t)\right)+g\left(F_{S T z, L x}(t)\right)\right)\right\}\right) \text {. }
\end{aligned}
$$

Letting $\mathrm{n} \rightarrow \infty$ and using equation (3.1.8) and Step 5, we get

$$
\begin{array}{r}
\mathrm{g}\left(\mathrm{F}_{\mathrm{z}, \mathrm{Mz}}(\mathrm{t})\right) \leq \phi\left(\operatorname { m a x } \left\{\mathrm{g}\left(\mathrm{F}_{\mathrm{z}, \mathrm{Mz}}(\mathrm{t})\right), \mathrm{g}\left(\mathrm{F}_{\mathrm{z}, \mathrm{z}}(\mathrm{t})\right), \mathrm{g}\left(\mathrm{F}_{\mathrm{Mz}, \mathrm{Mz}}(\mathrm{t})\right),\right.\right. \\
\left.\left.1 / 2\left(\mathrm{~g}\left(\mathrm{~F}_{\mathrm{z}, \mathrm{Mz}}(\mathrm{t})\right)+\mathrm{g}\left(\mathrm{F}_{\mathrm{Mz}, \mathrm{z}}(\mathrm{t})\right)\right)\right\}\right) \\
=\phi\left(\mathrm{g}\left(\mathrm{F}_{\mathrm{z}, \mathrm{Mz}}(\mathrm{t})\right)\right)
\end{array}
$$

which implies that $\mathrm{g}\left(\mathrm{F}_{\mathrm{z}, \mathrm{Mz}}(\mathrm{t})\right)=0$ by Lemma 2.1 and so we have $\mathrm{z}=\mathrm{Mz}$.

Step 7. Putting $x=x_{2 n}$ and $y=T z$ for $t>0$ in (3.1.5), we get

$$
\begin{aligned}
& \mathrm{g}\left(\mathrm{F}_{\mathrm{Lx}_{2 \mathrm{n}}, \mathrm{MTz}}(\mathrm{t})\right) \leq \phi\left(\operatorname { m a x } \left\{\mathrm{g}\left(\mathrm{F}_{\mathrm{ABx}_{2 \mathrm{n}}, \mathrm{STTz}}(\mathrm{t})\right), \mathrm{g}\left(\mathrm{F}_{\mathrm{ABx}_{2 \mathrm{n}}, \mathrm{Lx}_{2 \mathrm{n}}}(\mathrm{t})\right), \mathrm{g}\left(\mathrm{F}_{\mathrm{STTz}, \mathrm{MTz}}(\mathrm{t})\right)\right.\right. \text {, }
\end{aligned}
$$

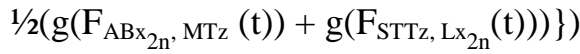

As $\mathrm{MT}=\mathrm{TM}$ and $\mathrm{ST}=\mathrm{TS}$ we have

$$
\mathrm{MTz}=\mathrm{TMz}=\mathrm{Tz} \quad \text { and } \quad \mathrm{ST}(\mathrm{Tz})=\mathrm{T}(\mathrm{STz})=\mathrm{Tz} .
$$

Letting $\mathrm{n} \rightarrow \infty$, we get 
Weakly Semi-Compatible Maps and Fixed Points in Non-Archimedean Menger PM-Space

$$
\begin{aligned}
\mathrm{g}\left(\mathrm{F}_{\mathrm{z}, \mathrm{Tz}}(\mathrm{t})\right) & \leq \phi\left(\operatorname { m a x } \left\{\mathrm{g}\left(\mathrm{F}_{\mathrm{z}, \mathrm{Tz}}(\mathrm{t})\right), \mathrm{g}\left(\mathrm{F}_{\mathrm{z}, \mathrm{z}}(\mathrm{t})\right), \mathrm{g}\left(\mathrm{F}_{\mathrm{Tz}, \mathrm{Tz}}(\mathrm{t})\right),\right.\right. \\
\left.\left.1 / 2\left(\mathrm{~g}\left(\mathrm{~F}_{\mathrm{z}, \mathrm{Tz}}(\mathrm{t})\right)+\mathrm{g}\left(\mathrm{F}_{\mathrm{Tz}, \mathrm{z}}(\mathrm{t})\right)\right)\right\}\right) & \\
& =\phi\left(\mathrm{g}\left(\mathrm{F}_{\mathrm{z}, \mathrm{Tz}}(\mathrm{t})\right)\right),
\end{aligned}
$$

which implies that $\mathrm{g}\left(\mathrm{F}_{\mathrm{z}, \mathrm{Tz}}(\mathrm{t})\right)=0$ by Lemma 2.1 and so we have $\mathrm{z}=\mathrm{Tz}$.

Now $\quad \mathrm{STz}=\mathrm{Tz}=\mathrm{z}$ implies $\mathrm{Sz}=\mathrm{z}$.

(3.1.10) Hence $\mathrm{Sz}=\mathrm{Tz}=\mathrm{Mz}=\mathrm{z}$.

Combining (3.1.9) and (3.1.10), we get

$$
\mathrm{Az}=\mathrm{Bz}=\mathrm{Lz}=\mathrm{Mz}=\mathrm{Tz}=\mathrm{Sz}=\mathrm{z} .
$$

Hence, the six self maps have a common fixed point in this case.

Case II. $\mathrm{L}$ is continuous.

As $\mathrm{L}$ is continuous, $\mathrm{L}^{2} \mathrm{x}_{2 \mathrm{n}} \rightarrow \mathrm{Lz}$ and $\mathrm{L}(\mathrm{AB}) \mathrm{x}_{2 \mathrm{n}} \rightarrow \mathrm{Lz}$.

Step 8. Putting $x=\operatorname{Lx}_{2 n}$ and $y=x_{2 n+1}$ for $t>0$ in (3.1.5), we get

$$
\begin{aligned}
& \mathrm{g}\left(\mathrm{F}_{\mathrm{LLx}_{2 n}, \mathrm{Mx} x_{2 \mathrm{n}+1}}(\mathrm{t})\right) \leq \phi\left(\operatorname { m a x } \left\{\mathrm{g}\left(\mathrm{F}_{\mathrm{ABLx}}, \mathrm{ST} \mathrm{Tx}_{2 \mathrm{n}+1}(\mathrm{t})\right), \mathrm{g}\left(\mathrm{F}_{\mathrm{ABLx} \mathrm{x}_{2 n}, \mathrm{LLx}_{2 \mathrm{n}}}(\mathrm{t})\right)\right.\right. \text {, } \\
& \mathrm{g}\left(\mathrm{F}_{\mathrm{STx}_{2 \mathrm{n}+1}, \mathrm{M} \mathrm{x}_{2 \mathrm{n}+1}}(\mathrm{t})\right) \text {, } \\
& \left.\left.1 / 2\left(\mathrm{~g}\left(\mathrm{~F}_{\mathrm{ABLx} 2 \mathrm{n}}, \mathrm{Mx}_{2 \mathrm{n}+1}(\mathrm{t})\right)+\mathrm{g}\left(\mathrm{F}_{\mathrm{STx}_{2 \mathrm{n}+1}, \operatorname{LLx}_{2 \mathrm{n}}}(\mathrm{t})\right)\right)\right\}\right) \text {. }
\end{aligned}
$$

Letting $\mathrm{n} \rightarrow \infty$, we get

$$
\begin{aligned}
\mathrm{g}\left(\mathrm{F}_{\mathrm{Lz}, \mathrm{z}}(\mathrm{t})\right) \leq & \phi\left(\operatorname { m a x } \left\{\mathrm{g}\left(\mathrm{F}_{\mathrm{Lz}, \mathrm{z}}(\mathrm{t})\right), \mathrm{g}\left(\mathrm{F}_{\mathrm{Lz}, \mathrm{Lz}}(\mathrm{t})\right), \mathrm{g}\left(\mathrm{F}_{\mathrm{z}, \mathrm{z}}(\mathrm{t})\right),\right.\right. \\
\left.\left.1 / 2\left(\mathrm{~g}\left(\mathrm{~F}_{\mathrm{Lz}, \mathrm{z}}(\mathrm{t})\right)+\mathrm{g}\left(\mathrm{F}_{\mathrm{z}, \mathrm{Lz}}(\mathrm{t})\right)\right)\right\}\right) & =\phi\left(\mathrm{g}\left(\mathrm{F}_{\mathrm{Lz}, \mathrm{z}}(\mathrm{t})\right)\right),
\end{aligned}
$$

which implies that $\mathrm{g}\left(\mathrm{F}_{\mathrm{Lz}, \mathrm{z}}(\mathrm{t})\right)=0$ by Lemma 2.1 and so we have $\mathrm{Lz}=\mathrm{z}$.

Now, using steps 5-7 gives us $\mathrm{Mz}=\mathrm{STz}=\mathrm{Sz}=\mathrm{Tz}=\mathrm{z}$.

Step 9. As $M(X) \subset A B(X)$, there exists $w \in X$ such that $z=M z=A B w$.

Putting $\mathrm{x}=\mathrm{w}$ and $\mathrm{y}=\mathrm{x}_{2 \mathrm{n}+1}$ for $\mathrm{t}>0$ in (3.1.5), we get

$$
\begin{aligned}
& \mathrm{g}\left(\mathrm{F}_{\mathrm{Lw}^{\prime}, \mathrm{Mx}} x_{2 \mathrm{n}+1}(\mathrm{t})\right) \leq \phi\left(\operatorname { m a x } \left\{\mathrm{g}\left(\mathrm{F}_{\mathrm{ABw}, \mathrm{ST} x_{2 n+1}}(\mathrm{t})\right), \mathrm{g}\left(\mathrm{F}_{\mathrm{ABw}, \mathrm{Lw}}(\mathrm{t})\right), \mathrm{g}\left(\mathrm{F}_{\mathrm{ST}_{2 n+1}, \mathrm{Mx}_{2 n+1}}(\mathrm{t})\right)\right.\right. \text {, } \\
& \left.\left.1 / 2\left(g\left(F_{A B w}, M_{2 n+1}(t)\right)+g\left(F_{S^{2} x_{2 n+1}, L w}(t)\right)\right)\right\}\right) .
\end{aligned}
$$

Letting $\mathrm{n} \rightarrow \infty$, we get

$$
\begin{aligned}
\mathrm{g}\left(\mathrm{F}_{\mathrm{Lw}, \mathrm{Z}}(\mathrm{t})\right) & \leq \phi\left(\operatorname { m a x } \left\{\mathrm{g}\left(\mathrm{F}_{\mathrm{z}, \mathrm{z}}(\mathrm{t})\right), \mathrm{g}\left(\mathrm{F}_{\mathrm{z}, \mathrm{Lw}}(\mathrm{t})\right), \mathrm{g}\left(\mathrm{F}_{\mathrm{z}, \mathrm{z}}(\mathrm{t})\right),\right.\right. \\
\left.\left.1 / 2\left(\mathrm{~g}\left(\mathrm{~F}_{\mathrm{z}, \mathrm{z}}(\mathrm{t})\right)+\mathrm{g}\left(\mathrm{F}_{\mathrm{z}, \mathrm{Lw}}(\mathrm{t})\right)\right)\right\}\right) & \\
& =\phi\left(\mathrm{g}\left(\mathrm{F}_{\mathrm{Lw}, \mathrm{z}}(\mathrm{t})\right)\right),
\end{aligned}
$$

which implies that $g\left(F_{L w, z}(t)\right)=0$ by Lemma 2.1 and so we have $L w=z$.

Thus, we have $\mathrm{Lw}=\mathrm{z}=\mathrm{ABw}$.

As $(\mathrm{L}, \mathrm{AB})$ is semi-compatible, so $\mathrm{L}(\mathrm{AB}) \mathrm{x}_{2 \mathrm{n}} \rightarrow \mathrm{ABz}$ and

as $\mathrm{L}$ is continuous, $\mathrm{L}^{2} \mathrm{x}_{2 \mathrm{n}} \rightarrow \mathrm{Lz}$ and $\mathrm{L}(\mathrm{AB}) \mathrm{x}_{2 \mathrm{n}} \rightarrow \mathrm{Lz}$.

Since limit of a sequence is unique, so $\mathrm{ABz}=\mathrm{Lz}$.

Also, $\mathrm{Bz}=\mathrm{z}$ follows from step 4 .

Thus, $\mathrm{Az}=\mathrm{Bz}=\mathrm{Lz}=\mathrm{z}$ and we obtain that $\mathrm{z}$ is the common fixed point of the six maps in this case also.

Step 10. (Uniqueness) Let $\mathrm{u}$ be another common fixed point of $\mathrm{A}, \mathrm{B}, \mathrm{S}, \mathrm{T}, \mathrm{L}$ and $\mathrm{M}$; then $\mathrm{Au}=\mathrm{Bu}=\mathrm{Su}=\mathrm{Tu}=\mathrm{Lu}=\mathrm{Mu}=\mathrm{u}$.

Putting $\mathrm{x}=\mathrm{z}$ and $\mathrm{y}=\mathrm{u}$ for $\mathrm{t}>0$ in (3.1.5), we get 
V .K. Gupta, Arihant Jain and Rambabu Dangi

$$
\begin{array}{r}
\mathrm{g}\left(\mathrm{F}_{\mathrm{Lz}, \mathrm{Mu}}(\mathrm{t})\right) \leq \phi\left(\operatorname { m a x } \left\{\mathrm{g}\left(\mathrm{F}_{\mathrm{ABz}, \mathrm{STu}}(\mathrm{t})\right), \mathrm{g}\left(\mathrm{F}_{\mathrm{ABz}, \mathrm{Lz}}(\mathrm{t})\right), \mathrm{g}\left(\mathrm{F}_{\mathrm{STu}, \mathrm{Mu}}(\mathrm{t})\right),\right.\right. \\
\left.\left.1 / 2\left(\mathrm{~g}\left(\mathrm{~F}_{\mathrm{ABz}, \mathrm{Mu}}(\mathrm{t})\right)+\mathrm{g}\left(\mathrm{F}_{\mathrm{STu}, \mathrm{Lz}}(\mathrm{t})\right)\right)\right\}\right) .
\end{array}
$$

Letting $\mathrm{n} \rightarrow \infty$, we get

$$
\begin{aligned}
\mathrm{g}\left(\mathrm{F}_{\mathrm{z}, \mathrm{u}}(\mathrm{t})\right) & \leq \phi\left(\max \left\{\mathrm{g}\left(\mathrm{F}_{\mathrm{z}, \mathrm{u}}(\mathrm{t})\right), \mathrm{g}\left(\mathrm{F}_{\mathrm{z}, \mathrm{z}}(\mathrm{t})\right), \mathrm{g}\left(\mathrm{F}_{\mathrm{u}, \mathrm{u}}(\mathrm{t})\right), 1 / 2\left(\mathrm{~g}\left(\mathrm{~F}_{\mathrm{z}, \mathrm{u}}(\mathrm{t})\right)+\mathrm{g}\left(\mathrm{F}_{\mathrm{u}, \mathrm{z}}(\mathrm{t})\right)\right)\right\}\right) \\
& =\phi\left(\mathrm{g}\left(\mathrm{F}_{\mathrm{z}, \mathrm{u}}(\mathrm{t})\right)\right),
\end{aligned}
$$

which implies that $\mathrm{g}\left(\mathrm{F}_{\mathrm{z}, \mathrm{u}}(\mathrm{t})\right)=0$ by Lemma 2.1 and so we have $\mathrm{z}=\mathrm{u}$.

Therefore, $\mathrm{z}$ is a unique common fixed point of $\mathrm{A}, \mathrm{B}, \mathrm{S}, \mathrm{T}, \mathrm{L}$ and $\mathrm{M}$.

This completes the proof.

Remark 3.1. If we take $B=T=I$, the identity map on $X$ in theorem 3.1, then the condition (3.1.2) is satisfied trivially and we get

Corollary 3.1. Let A, S, L, $M: X \rightarrow X$ be mappings satisfying the condition :

(3.1.11) $\mathrm{L}(\mathrm{X}) \subseteq \mathrm{S}(\mathrm{X}), \quad \mathrm{M}(\mathrm{X}) \subseteq \mathrm{A}(\mathrm{X})$;

(3.1.12) Either $\mathrm{A}$ or $\mathrm{L}$ is continuous;

(3.1.13) the pair (L, A) is semi-compatible and (M, S) is occasionally weakly compatible;

(3.1.14) $g\left(F_{L x, M y}(t)\right) \leq \phi\left(\max \left\{g\left(F_{A x, S y}(t)\right), g\left(F_{A x}, L x(t)\right), g\left(F_{S y, M y}(t)\right)\right.\right.$,

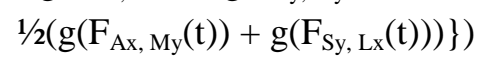

for all $\mathrm{t}>0$, where a function $\phi:[0,+\infty) \rightarrow[0,+\infty)$ satisfies the condition $(\Phi)$.

Then $\mathrm{A}, \mathrm{S}, \mathrm{L}$ and $\mathrm{M}$ have a unique common fixed point in $\mathrm{X}$.

Remark 3.2. In view of remark 3.1, corollary 3.1 is a generalization of the result of Cho et al. [2] and theorem 3.1 is a generalization of the result of Sharma et al. [14] in the sense that condition of compatibility of the pairs of self maps has been restricted to weakly semi-compatible and occasionally weakly compatible self maps and only one of the mappings of the first pair is needed to be continuous.

\section{REFERENCES}

1. S.S.Chang, Fixed point theorems for single-valued and multi-valued mappings in non-Archimedean Menger probabilistic metric spaces, Math. Japonica, 35(5) (1990) 875-885.

2. Y.J.Cho, K.S.Ha and S.S.Chang, Common fixed point theorems for compatible mappings of type (A) in non-Archimedean Menger PM-spaces, Math. Japonica, 48(1) (1997) 169-179.

3. Y.J.Cho, K.S.Park and S.S.Chang, Fixed point theorems in metric spaces and probabilistic metric spaces, Internat. J. Math. \& Math. Sci., 19(2) (1996) 243-252.

4. A.Jain and B.Singh, Common fixed point theorem in Menger space through compatible maps of type (A), Chh. J. Sci. Tech., 2 (2005) 1-12.

5. A.Jain and B.Singh, A fixed point theorem in Menger space through compatible maps of type (A), V.J.M.S., 5(2) (2005) 555-568.

6. A.Jain and B.Singh, Common fixed point theorem in Menger Spaces, The Aligarh Bull. of Math., 25(1) (2006) 23-31. 
Weakly Semi-Compatible Maps and Fixed Points in Non-Archimedean Menger

PM-Space

7. O.Hadzic, A note on Istră trescu's fixed point theorems in non-Archimedean Menger spaces, Bull. Math. Soc. Sci. Math. Rep. Soc. Roum. T., 24(72) (1980) 277-280.

8. V.I.Istra tescu, Fixed point theorems for some classes of contraction mappings on nonarchimedean probabilistic metric space, Publ. Math. (Debrecen), 25 (1978) 2934.

9. V.I.Istră tescu and N.Crivat, On some classes of nonarchimedean Menger spaces, Seminar de spatii Metrice probabiliste, Univ. Timisoara Nr. 12 (1974).

10. K.Menger, Statistical metrics, Proc. Nat. Acad. Sci., 28 (1942) 535-537.

11. S.N.Mishra, Common fixed points of compatible mappings in PM-spaces, Math. Japon., 36(2) (1991) 283-289.

12. B.Schweizer and A.Sklar, Statistical metric spaces, Pacific J. Math., 10 (1960) 313334.

13. V.M.Sehgal and A.T.Bharucha-Reid, Fixed points of contraction maps on probabilistic metric spaces, Math. System Theory, 6 (1972) 97- 102.

14. A.Sharma, A.Jain and S.Choudhari, Occasionally weakly compatible maps and fixed points in Non-Archimedean Menger PM-space, J. Nat. Acad. Math., 25 (2011) 25-36.

15. B.Singh, A.Jain and P.Agarwal, Weak-compatibility in non-archimedean Menger PM-Space, Journal of Indian Acad. Math., 31(2) (2009) 335-350.

16. B.Singh, A.Jain and P.Agarwal, Semi-compatibility in Non-Archimedean Menger PM-Space, Commentationes Mathematicae, 49(1) (2009) 15-25. 